\title{
Smoothed Particle Hydrodynamics - partikelbasierte Strömungsberechnung zur Anwendung in der Siedlungswasserwirtschaft
}

\author{
Michael Meister · Nikolaus Fleischhacker · Wolfgang Rauch \\ Online publiziert: 10. April 2015 \\ (c) Die Autor(en) 2015. Dieser Artikel ist auf Springerlink.com mit Open Access verfügbar
}

Zusammenfassung Zahlreiche Strömungsprobleme in der Siedlungswasserwirtschaft können mittels vereinfachter Methoden berechnet werden. Zum Beispiel werden Reaktoren meist als voll durchmischt angenommen und Gerinneströmungen durch ein eindimensionales Modell approximiert. Beeinflusst aber die Strömung die Funktion der Infrastruktur, ist es notwendig, komplexere numerische Berechnungen mit Computational-Fluid-Dynamics-Methoden durchzuführen. Beispiele dafür sind die Modellierung von Absetzbecken, Faulbehältern und komplexen Mischwasserbehandlungsanlagen. Numerische Strömungsberechnungen in der Siedlungswasserwirtschaft verwenden bislang gitterbasierte Computational-Fluid-Dynamics-Methoden. In diesem Artikel wird anhand von Beispielen die Anwendungsmöglichkeit einer alternativen partikelbasierten Methode, nämlich von Smoothed Particle Hydrodynamics, diskutiert.

\section{Smoothed particle} hydrodynamics: particle-based fluid dynamics calculations for use in sanitary engineering

Abstract Many of the fluid dynamics problems involved in sanitary engineering can be calculated with the help of simplified methods. For example, reactors are most often assumed to be fully mixed and open-channel flows are approximated using a one-dimensional model. If the flows influence the function of the infrastructure, however, it becomes necessary to carry out more complex

\footnotetext{
M. Meister, BSc, MSc ( $\square)$.

DI N. Fleischhacker .

Univ.-Prof. DI Dr. W. Rauch

Arbeitsbereich für Umwelttechnik, Institut für Infrastruktur, Universität Innsbruck, Technikerstraße 13,

6020 Innsbruck, Österreich

E-Mail: Michael.Meister@uibk.ac.at
}

numerical calculations using Computational Fluid Dynamics (CFD) methods; examples include the modeling of sedimentation tanks, digesters and complex mixed water treatment facilities. To date, numerical calculations in sanitary engineering have used grid-based CFD methods. The following article discusses the potential applications of an alternative particle-based method, Smoothed Particle Hydrodynamics, on the basis of practical examples.

\section{Einleitung}

Im weitesten Zusammenhang beschäftigt sich Siedlungswasserwirtschaft mit dem Transport und der Umsetzung von Wasser und dessen Inhaltsstoffen im urbanen Kontext. Wasserströmungen kommen daher in fast allen Teilen des realen Systems vor. Es ist aber vielfach möglich diese Strömungen mittels des Modells eines Reaktors stark vereinfacht zu beschreiben (z. B. in Kläranlagen, siehe Levenspiel 1972) oder das 3-dimensionale Problem auf eine eindimensionale Form zu reduzieren. Die eindimensionale Strömungsberechnung in Form der St.-Venant-Gleichungen wird vor allem in der Kanalnetzberechnung eingesetzt (Rauch et al. 2010). Es gibt jedoch vereinzelt Problemstellungen, die eine detaillierte numerische Strömungsberechnung mittels Computational Fluid Dynamics (CFD) erfordern. Beispiele hierfür sind die Modellierung von Absetzbecken (Brouckaert und Buckley 1999), Regenüberläufen (Fach et al. 2009) und Faulbehältern (Wu 2010). In diesen Berechnungen werden standardmäßig gitterbasierte CFD-Methoden eingesetzt.

Vor etwa 3 Jahrzehnten wurde im Umfeld der Astrophysik eine alternative Strömungsberechnungsmethode entwickelt, nämlich Smoothed Particle Hydrodynamics (SPH) (Sitzenfrei et al. 2014b). Dabei wird die Flüssigkeit durch eine Vielzahl von Partikeln gleicher Masse beschrieben, sodass die Strömung durch die (freie) Bewegung der Partikel ausgedrückt bzw. berechnet wird. Dies bedingt einen hohen Rechenaufwand, da die Bewegung jedes einzelnen Partikels berechnet werden muss, sodass dynamische Strömungsvorgänge nur über kurze Zeiträume betrachtet werden können. Andererseits ist die Berechnungsmethode sehr flexibel und erlaubt die Einführung komplexer Randbedingungen sowie die Simulation von Mehrphasenströmungen. Aufgrund dieser Vorteile wird SPH zwischenzeitlich vielfach in der Industrie verwendet.

Ziel dieser Arbeit ist es, die Stärken und Schwächen der SPH-Methode für Anwendungen in der Siedlungswasserwirtschaft vorzustellen. Anstelle einer komplexen vollständigen Beschreibung der Methodik und der detaillierten Abhandlung eines Einzelbeispiels wird der Schwerpunkt auf einen Gesamtüberblick gesetzt. Wir führen bewusst eine Vielzahl einfacher Anwendungsbeispiele zur Simulation von Gerinneströmungen, Sedimentations- und Transportvorgängen bzw. Belüftungsprozessen in der Abwasserreinigung an und verdeutlichen jeweils die Vor- und Nachteile von SPH. In einem abschließenden Kapitel werden die Anwendbarkeit auf reale Probleme und der damit verbundene Rechenaufwand diskutiert.

\section{Methodik}

Das Grundprinzip dieses Ansatzes beruht darauf, eine Flüssigkeit durch Diskretisierung in Partikel konstanter Masse zu modellieren. Jedem SPH-Partikel werden physikalische Parameter wie Dichte, Druck und Geschwindigkeit zugeordnet, deren Zeitentwicklung durch ein geschlossenes Differenzialgleichungssystem von Kontinuitäts-, Impuls- und Zustandsgleichung beschrieben und durch Gewichtung der Nachbarschaft gelöst wird (vgl. Abb. 1). Das heißt, die Änderung der Dichte bzw. der Geschwindigkeit eines Referenzparti- 

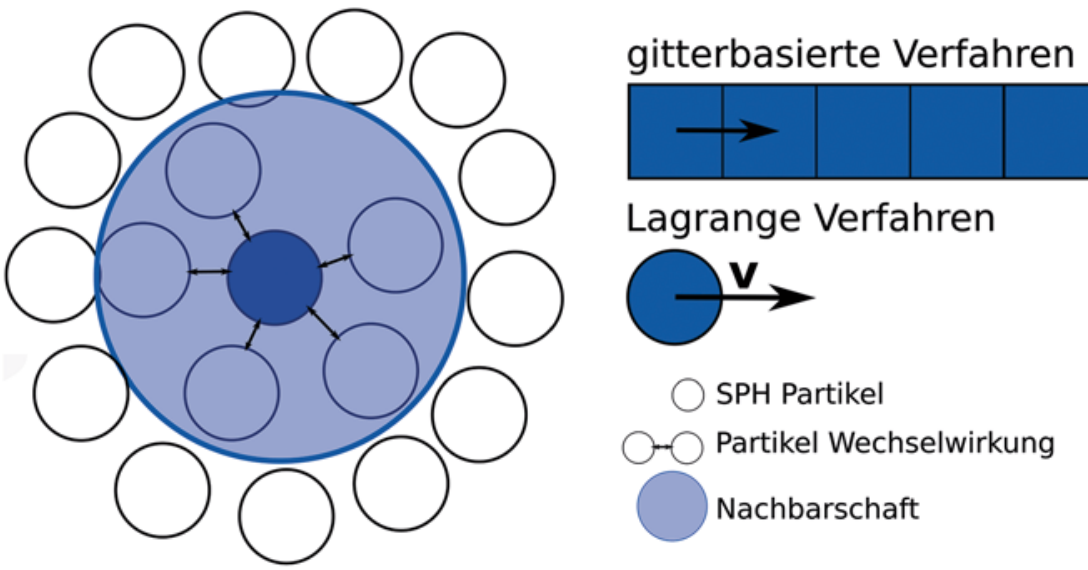

\section{SPH Partikel}

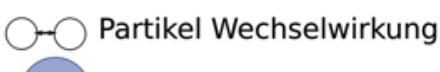

Nachbarschaft

Abb. 1 Advektion eines SPH-Partikels durch Gewichtung des Einflusses von 5 Nachbarn. (abgeänderte Grafik aus Sitzenfrei et al. 2014b)

kels wird anhand der Parameter der Nachbarpartikel berechnet, sodass anstelle einer partiellen Differenzialgleichung ein System gewöhnlicher Differenzialgleichungen numerisch gelöst wird.

Die Verwendung von SPH in der Siedlungswasserwirtschaft hat mehrere Vorteile im Vergleich zu konventionellen gitterbasierten CFD-Methoden. Da jedes Partikel eine konstante Masse trägt und die Anzahl konstant bleibt, ist die Massenbilanz implizit erfüllt. Zudem führt die Berechnung des Druckes durch Gewichtung der Nachbarpartikel zu einer Nulldruckbedingung an Wasseroberflächen, sodass die Simulation freier Oberflächen direkt unterstützt wird. Weiters ermöglicht die Standardformulierung von SPH Mehrphasensimulationen, wobei bei hohen Dichteunterschieden wie z. B. bei Luft-Wasser-Interaktionen, numerische Korrekturen notwendig sind (Colagrossi und Landrini 2003; Monaghan 2013). Aufgrund der exakten Beschreibung der Advektion ist SPH besonders zur Simulation von Transportvorgängen in Gerinnen geeignet. Durch Markierung einzelner Partikel kann deren Bahnlinie verfolgt werden. Abschließend ist die Geometrie eines Pro- blems sofort adaptierbar, da nur die initiale Partikelverteilung verändert werden muss. Insbesondere werden feste Ränder durch stationäre Partikel beschrieben, wobei ein Kräftegleichgewicht zwischen Flüssigkeits- und stationären Wandpartikeln angesetzt wird (Adami et al. 2012).

\section{Anwendungsbeispiele}

\subsection{Gerinneströmungen}

Die Modellierung von Gerinneströmungen mit SPH hat den Vorteil, dass sowohl ein Rückstau, Zuflüsse z. B. durch Niederschlagswasser als auch Transportvorgänge beschrieben werden können. Aufgrund der Notwendigkeit einer detailgetreuen Auflösung wird jedoch schon bei niedrigen Reynoldszahlen ein Turbulenzmodell zur Erreichung korrekter Geschwindigkeitsprofile und zur Sicherstellung einer Langzeitstabilität benötigt (Meister et al. 2014). Nur in Spezialfällen wie z. B. bei einem laminaren Poiseuille- bzw. Couette-Fluss für geschlossene Gerinne liefert die Standardformulierung auch bei geringer Auflösung akkurate Ergebnisse (vgl. Abb. 2).
Bei Abweichungen von diesen einfachen laminaren Strömungsprofilen, wie zum Beispiel beim Einstau durch ein Wehr, ist eine detailliertere Auflösung durch Erhöhung der Partikelanzahl notwendig. Exemplarisch zeigt Abb. 3 die SPH-Simulation eines Poleni-Überfalls bzw. eines Torricelli-Ausflusses. Die Überprüfung des simulierten Durchflusses mit dem nach der Poleni-Überfallformel berechneten Wert

$$
Q_{S P H}=1.675 \pm 0.045 \frac{\mathrm{m}^{2}}{\mathrm{~s}} \approx 1.661 \frac{\mathrm{m}^{2}}{\mathrm{~s}}=Q_{\text {Poleni }}
$$

zeigt eine weitgehende Übereinstimmung (Meister et al. 2014).

\subsubsection{Eindimensionale Näherung}

Um vollständige Kanalnetze simulieren zu können, werden Gerinne in der Urbanhydrologie meist durch eindimensionale Näherungen beschrieben. Falls der Einfluss der lokalen Beschleunigung und Höhenänderung vernachlässigt wird, können die Flachwassergleichungen durch den kinematischen Wellenansatz beschrieben werden (Ponce 1991). In der Siedlungswasserwirtschaft hat sich zur Simulation von Kanalnetzen u. a. das Softwarepaket SWMM (StormWater Management Model) etabliert, welches auf dem eulerbasierten Finite-Differenzen-Verfahren zur örtlichen und zeitlichen Diskretisierung basiert.

Unter der Annahme des kinematischen Wellenansatzes haben wir einen neuen Flachwassergleichungs-SPH-Löser entwickelt (Fleischhacker et al. 2014). Im Vergleich zum in SWMM angewandten Finite-Differenzen-Verfahren gewährleistet der SPH-Löser die Massenbilanz und kann zur Beschreibung von Transportprozessen erweitert werden. Bei der neuen 1D-SPH-Formulierung wird die Partikelbewegung in Abhängigkeit von der Fluidhöhe h ermittelt, wobei über den Manning-Strickler-Reibungsansatz auf

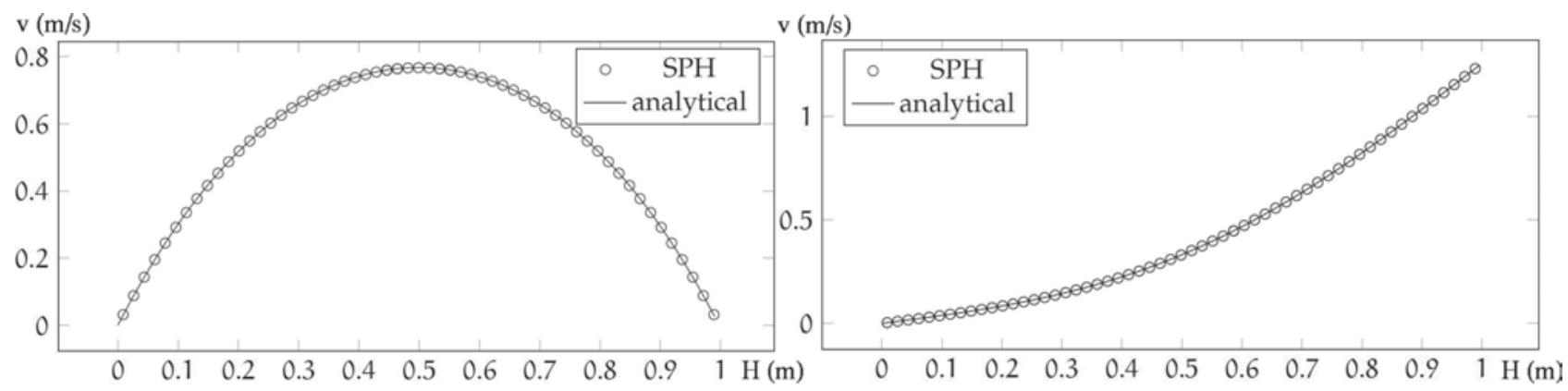

Abb. 2 Vergleich des SPH-Geschwindigkeitsprofils für den Poiseuille- und Couette-Fluss mit der analytischen Lösung 


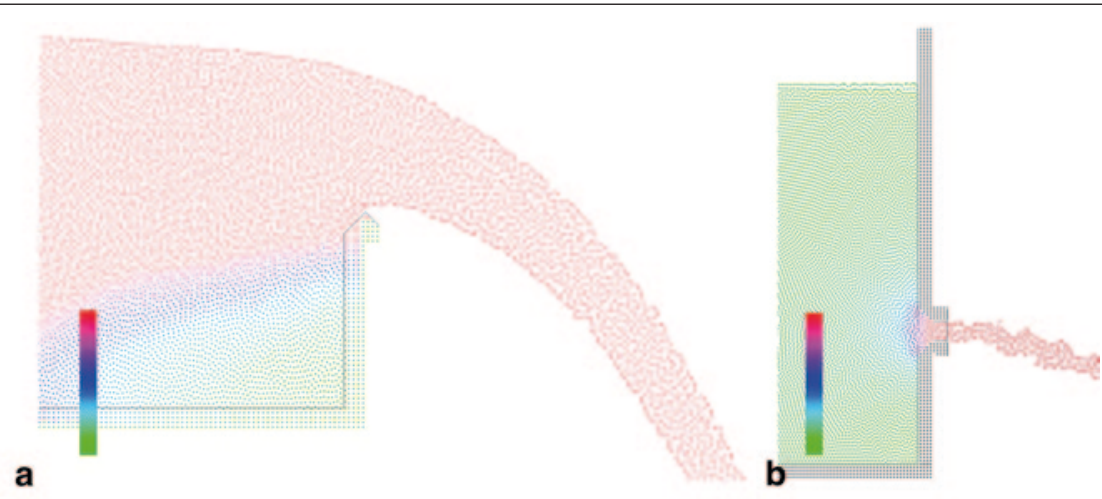

Abb. 3 Simulation eines Poleni-Überfalls bzw. eines Torricelli-Ausflusses mit SPH

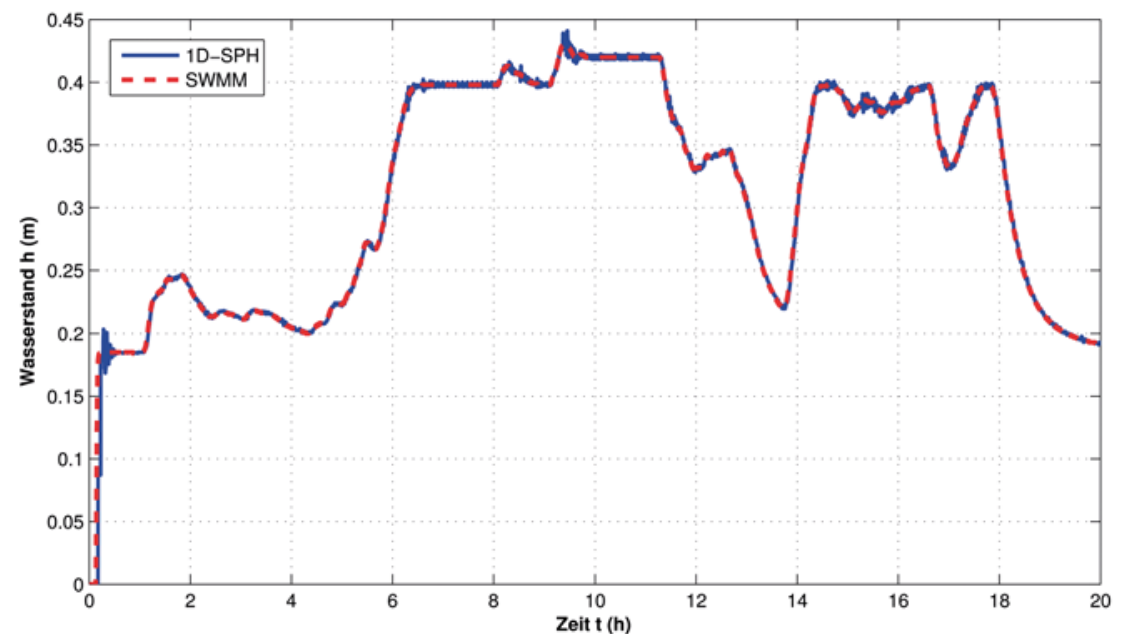

Abb. 4 Vergleich von SWMM mit einem neuen Algorithmus zur Simulation der kinematischen Welle mit SPH $(x=200 \mathrm{~m})$
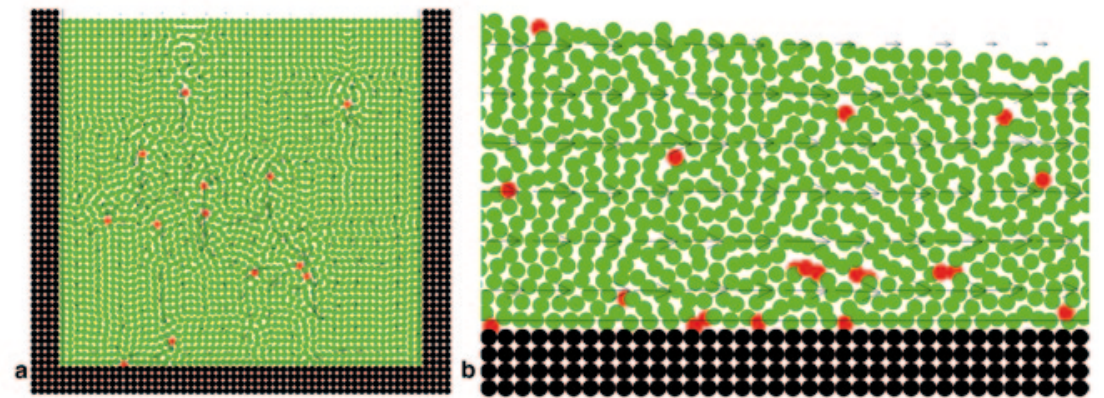

Abb. 5 Sedimentation und Geschwindigkeitsvektorfeld von Feststoffen mit der doppelten Dichte von Wasser in einem abgeschlossenen Becken (a) bzw. im Kanal (b)

die Partikelgeschwindigkeit v geschlossen wird.

Aufbauend auf die in Fleischhacker et al. (2014) präsentierten Ergebnisse, zeigen wir die Leistungsfähigkeit dieses Solvers am Beispiel der Berechnung einer Abflussganglinie in einem realen System (Sitzenfrei et al. 2014a). Die Abflussganglinie wird in beiden Implementierungen in 50 Abschnitte geteilt. Da in der SPHImplementierung die örtliche Auflösung adaptiv von der Partikelanzahl abhängt, ist die örtliche Auflösung bei hohen Wasserständen genauer als bei der SWMM Simulation. Der resultierende höhere Rechenaufwand kann durch die Einführung variabler Partikelvolumen in zukünftigen Implementierungen vermieden werden. Abbildung 4 zeigt eine gute Übereinstimmung der mit SWMM bzw. SPH simulierten Abflussganglinien. Die Genauigkeit der Simulationsergebnisse ist vergleichbar, der Rechenaufwand mit SPH ist aufgrund der teilweise genaueren Auflösung geringfügig höher.

\subsection{Sedimentations- und Transportvorgänge}

Im Unterschied zu den bisherigen Beispielen betrachten wir nachfolgend Mehrphasenprobleme, wobei bei der Wahl einer niedrigen Auflösung ein Feststoffpartikel durch ein einzelnes SPHPartikel beschrieben wird. Obwohl diese Näherung zu Interpolationsfehlern führt, sind diese bei geringen Dichteunterschieden wie im Fall Sediment-Wasser klein genug, damit die Simulationsmethode stabil ist und das Absetzverhalten korrekt beschrieben wird. Exemplarisch zeigt Abb. 5a die Sedimentation von Feststoffen mit der doppelten Dichte von Wasser und das dadurch induzierte Geschwindigkeitsvektorfeld in einem abgeschlossenen Becken. In Abb. 5b ist ein Kanalausschnitt $1 \mathrm{~m}$ unterwasserseitig eines konstanten Zuflusses mit einem Wasserstand von $0.5 \mathrm{~m}$ und einer Geschwindigkeit von $2 \mathrm{~m} / \mathrm{s}$ dargestellt. Im Zufluss werden gleichverteilt Feststoffpartikel mit der doppelten Dichte von Wasser eingetragen, welche sich unterwasserseitig zunehmend absetzen. Da mit dieser Methodik jedes Feststoffpartikel aufgelöst wird, ist der Rechenaufwand zu hoch, um längere Kanalsegmente (vgl. Abb. 4) bzw. komplexe Netze zu berechnen. Daher arbeiten wir daran, Transportvorgänge im Kanal auch mit dem eindimensionalen Flachwassergleichungs-SPH-Löser zu beschreiben bzw. eine Kopplung zwischen ein- und zweidimensionalen Modellen zu ermöglichen. Dadurch könnte die detaillierte Auflösung auf notwendige Bereiche reduziert werden. von $B=1 \mathrm{~m}$, einer Länge von $\mathrm{l}=1000 \mathrm{~m}$ und einer Gerinneneigung von $1 \% \mathrm{mit}$ einem Strickler-Beiwert von $k_{s t}=40 \frac{m^{1 / 3}}{}$ eingeleitet. Die zeitliche Diskretisierung wird in beiden Simulationen mit $\Delta t=1 \mathrm{~s}$ festgesetzt, wobei sich die örtliche Diskretisierung wie folgt unterscheidet: In SWMM wird der Kanal über die Länge

\subsubsection{Spülklappe}

Spülklappen dienen dem Transport sedimentierter Feststoffe in Kanälen mit geringer Neigung (bzw. geringer Sohl- 

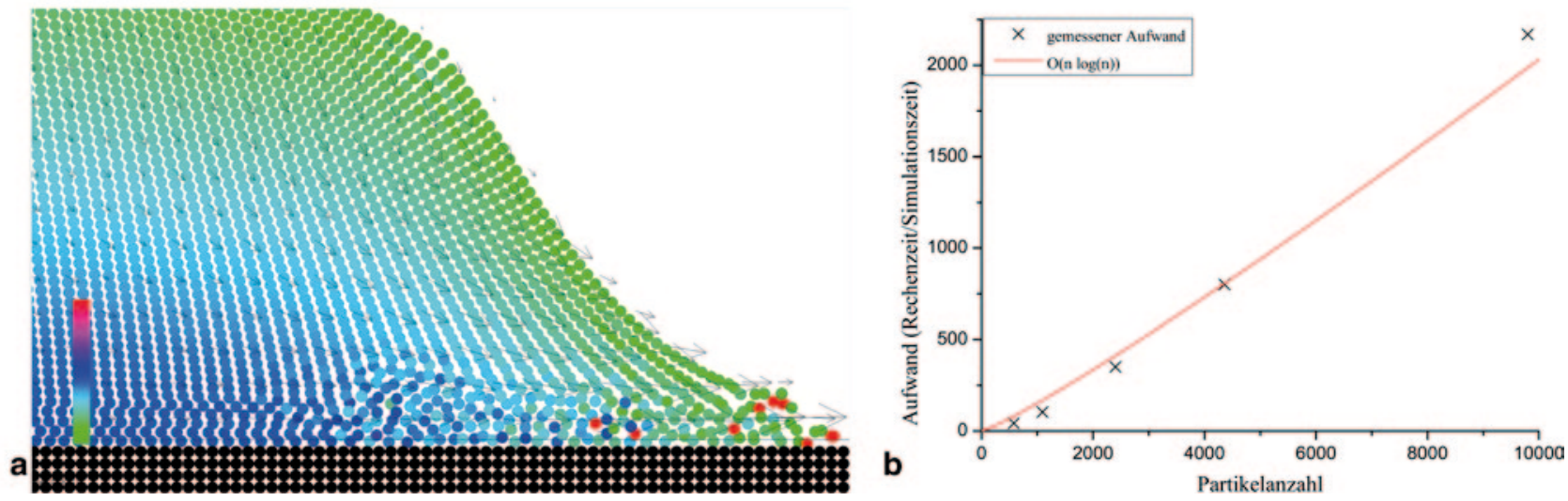

Abb. 6 Feststoffe mit der doppelten Dichte von Wasser werden in einer Spülklappe weggeschwemmt (a). In (b) ist der Rechenaufwand in Abhängigkeit von der Partikelanzahl (Auflösung) dargestellt

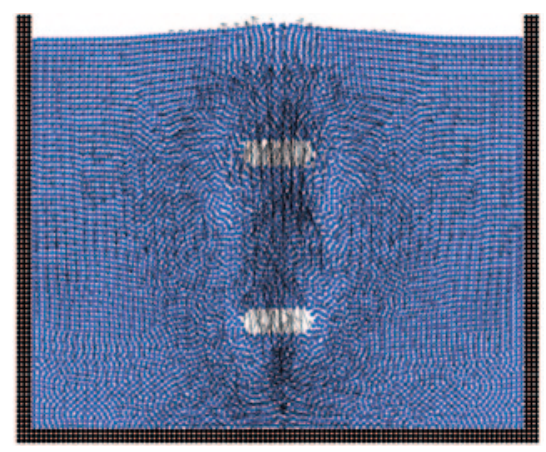

Abb. 7 Mehrskaliges SPH-Modell einer Luftsäule mit homogener Luftblasenverteilung. Eine Einzelblase wird durch $66 \mathrm{SPH}-$ Partikel mit einer experimentell vorgegebenen Aufstiegsgeschwindigkeit charakterisiert, sodass 5 min. physikalischer Zeit in $5 \mathrm{~h}$ simuliert werden können

schubspannung). Die komplexen Verhältnisse beim Herausschwemmen der Feststoffe in einer Spülklappe (vgl. Abb. 6) sind mit vereinfachten eindimensionalen Modellen nicht mehr abzubilden, sondern erfordern komplexere numerische Strömungsberechnungen (Schaffner 2008). Aufgrund der Simulation einer sich rasch deformierenden freien Oberfläche, welche sehr dynamisch mit abgesetzten Feststoffen interagiert, ist SPH besonders für dieses Anwendungsbeispiel geeignet. Insbesondere sind hierfür bereits detaillierte Modelle entwickelt worden: Sedimente werden dabei als schwach kompressible pseudo-newtonsche Flüssigkeit beschrieben und als Schwemm-Modell sind sowohl das MohrCoulomb- als auch das Drucker-PragerAblösemodell implementiert (Fourtakas et al. 2013).

\subsection{Belüftungsprozesse in der Abwasserreinigung}

In Ergänzung zur Beschreibung von Sedimentationsprozessen kann mit SPH in der Abwasserreinigung auch die Durchmischung im Belebungsbecken durch die eingeblasene Luft und der Sauerstofftransfer zwischen den verschiedenen Phasen durch advektive Diffusion (Aristodemo et al. 2010) simuliert werden. Allerdings ist die Standardformulation von SPH bei den vorherrschenden Dichteunterschieden von 1:1000 instabil. Entsprechende Korrekturen stabilisieren die Methode - z. B. durch geringfügige Erhöhung des Druckes an der Phasengrenze Luft-Wasser (Monaghan 2013) -, erhöhen aber den Rechenaufwand deutlich, sodass die Anwendung derzeit auf die Simulation von einzelnen Blasen (Abb. 7) limitiert ist (z. B. Meister und Rauch 2014). Nach detaillierter Auseinandersetzung mit der Anwendung von SPH in der Abwasserreinigung sehen wir die Stärke dieser Methode neben der Beschreibung eines dreiphasigen Modells (Luft-Wasser-Sediment) insbesondere in der Kopplung mit biokinetischen Modellen wie dem etablierten Activated-Sludge-Modell (ASM).

\section{Anwendbarkeit und Rechenaufwand}

Obwohl die dargestellten Anwendungsbeispiele nur Ausschnitte realer Problemstellungen darstellen, kann SPH direkt auf komplexere Systeme bzw. auf dreidimensionale Probleme angewendet werden. Wie bei allen partikelbasierten CFD-Methoden ist der Rechenaufwand hoch und für Anwendungen in der Siedlungswasserwirtschaft meist der limitie- rende Faktor. Abbildung 6b zeigt die für die Spülklappen-Simulation unter Verwendung eines 4-Kern-Bürocomputers bei Berücksichtigung von 28 Nachbarpartikeln benötigte Rechenzeit. Bei der niedrigsten Auflösung von $n=576$ Flüssigkeitspartikeln wird $1 \mathrm{~s}$ physikalische Zeit in $42 \mathrm{~s}$ berechnet. Dieser Wert steigt auf 2170s, wenn eine detailliertere Auflösung von $n=9800$ Partikel gewählt wird. Insbesondere skaliert der Rechenaufwand mit $\mathrm{O}(n \log (n))$, welches der (optimalen) Komplexität der Nachbarschaftssuche entspricht. Dieser Aufwand erlaubt zwar die detaillierte Berechnung kleinskaliger, abgeschlossener Systeme, die detaillierte Auflösung einer gesamten Kläranlage bzw. von großen Kanalnetzen ist aber - mit Ausnahme des eindimensionalen Flachwassergleichungs-Lösers - mit den zurzeit verfügbaren Computerressourcen noch nicht möglich. Nichtsdestotrotz ist die mathematische Struktur von SPH hochgradig parallel und die Berechnung wird zunehmend auf Grafikkarten ausgelagert, sodass mittlerweile mit bis $\mathrm{zu}$ 100 Millionen. Partikeln gerechnet werden kann (Herault et al. 2010). Insgesamt zeigt die Methode ein hohes Potenzial für die Lösung von Spezialproblemen und kann dafür bereits heute vorteilhaft eingesetzt werden. Als generell anwendbares Werkzeug zur numerischen Strömungsberechnung ist die Methode derzeit noch zu rechenintensiv.

\section{Zusammenfassung}

Spezielle Problemstellungen in der Siedlungswasserwirtschaft erfordern komplexe Strömungsberechnungen, wobei dafür meist gitterbasierte CFDMethoden verwendet werden. Im Vergleich dazu bringt der hier präsentierte 


\section{innovative SPH-Ansatz zahlreiche} Vorteile mit sich. Wir haben die vielfältigen Einsatzmöglichkeiten in der Siedlungswasserwirtschaft zur Simulation von Gerinneströmungen, Sedimentations- und Transportvorgängen bzw. Belüftungsprozessen in der Abwasserreinigung anhand einfacher Beispiele aufgezeigt, sind uns aber bewusst, dass für die Etablierung von SPH in der Siedlungswasserwirtschaft noch einiges an Entwicklungsarbeit zu leisten ist. Die jeweiligen Modelle sind auf reale dreidimensionale Probleme direkt anwendbar. Allerdings ist die detaillierte Modellie- rung komplexer Kanalnetze bzw. kompletter Kläranlagen aufgrund des hohen Rechenaufwands mit den derzeitigen Computer-Ressourcen nicht möglich. Eine Lösung hierfür sehen wir einerseits in einer variablen Auflösung durch die Kopplung zwischen ein- und zweidimensionalen Modellen. Andererseits ist die SPH-Methode gut für die Parallelisierung und Berechnung auf Grafikkarten geeignet, sodass Simulationen mit bis zu 100 Millionen Partikeln möglich sind. Für spezielle Probleme ist das Verfahren bereits heute vorteilhaft anwendbar.

\section{Danksagung}

Die Forschungsergebnisse dieser Arbeit sind Teil des folgenden Projekts [P26768-N28], welches vom österreichischen Wissenschaftsfonds (FWF) gefördert wird.

Open Access Dieser Artikel unterliegt den Bedingungen der Creative Commons Attribution License. Dadurch sind die Nutzung, Verteilung und Reproduktion erlaubt, sofern der/die Originalautor/en und die Quelle angegeben sind.

\section{Literatur}

Adami, S., Hu, X., Adams, N. (2012): A generalized wall boundary condition for smoothed particle hydrodynamics. J. Comput. Phys. 231, 7057-7075

Aristodemo, F., Federico, I., Veltri, P., Panizzo, A. (2010): Two-phase SPH modelling of advective diffusion processes. Environ. Fluid Mech. 10, $451-470$.

Brouckaert, C., Buckley, C. (1999): The use of computational fluid dynamics for improving the design and operation of water and wastewate treatment plants. Water Science and Technology 40 (4-5), 81-89.

Colagrossi, A., Landrini, M. (2003): Numerical simulation of interfacial flows by smoothed particle hydrodynamics. J. Comput. Phys. 191, 448-475.

Fach, S., Sitzenfrei, R., Rauch, W. (2009): Determining the spill flow discharge of combined sewer overflows using rating curves based on computational fluid dynamics instead of the standard weir equation. Water Science and Technology 60 (12), 3035-3043
Fleischhacker, N., Burger, G., Meister, M. Rauch, W. (2014): Simulating kinematic wave with smoothed particle hydrodynamics. J. Hydroinform., under review in 2014

Fourtakas, G., Rogers, B. D., Laurence, D. (2013) Modelling sediment resuspension in industria tanks using SPH on GPUs. Proc. Int. Conf. SPHERIC SPH workshop Trondheim, 310-316. Herault, A., Bilotta, G., Dalrymple, R. A. (2010) SPH on GPU with CUDA. J. Hydraul. Res. 48, 74-79. Levenspiel, 0. (1972): Chemical Reaction Engineering. Wiley.

Meister, M., Rauch, W. (2014): Simulating rising bubble problems with smoothed particle hydrodynamics. Proc. Conf. IMA Mathematical Modelling of Fluid Systems Bristol, 1-10.

Meister, M., Burger, G., Rauch, W. (2014): On the Reynolds number sensitivity of smoothed particle hydrodynamics. J. Hydraul. Res. 52, 1-12.

Monaghan, J. (2013): A simple SPH algorithm for multi-fluid flow with high density ratios. Int. $J$. Numer. Meth. Fluids 71, 537-561.
Ponce, V. (1991): The kinematic wave controversy. J. Hydraul. Eng. 117, 511-525.

Rauch, W., Kleidorfer, M., Fach, S. (2010): Vom Bleistift zum Prozessor: Wandel der Modelle in der Siedlungsentwässerung. Österreichische Wasserund Abfallwirtschaft 62 (3-4), 43-50.

Schaffner, J. (2008): Numerical investigations on the function of flush waves in a reservoir sewer. Ph.D. thesis, TU Darmstadt, Germany.

Sitzenfrei, R., Kleidorfer, M., Arming, G. Möderl, M., Rauch, W. (2014a): Auswirkungen von alpinen urbanen Einzugsgebieten auf den Hochwasserschutz. Proc. Conf. Aqua Urbanica Innsbruck.

Sitzenfrei, R., Kleidorfer, M., Meister, M., Burger, G, Urich, C., Mair, M., Rauch, W. (2014b): Scientific computing in urban water management. In Hofstetter, G. (Ed.), Computational Engineering. Springer International Publishing, pp. 173-193.

Wu, B. (2010): CFD simulation of mixing in eggshaped anaerobic digesters. Water Research 44 (5), 1507-1519. 